\title{
Successful integration of ergonomics into continuous improvement initiatives
}

\author{
Kimberly Monroe ${ }^{\mathrm{a}}$, Faye Fick ${ }^{\mathrm{b}}$ and Madina Joshi ${ }^{\mathrm{c}}$ \\ ${ }^{a}$ KM Ergonomics, LLC, PO Box 4920, Boulder, CO 80306, USA \\ ${ }^{\mathrm{b}}$ Worksite Therapies and Consulting Services, PO Box, 210 Virginville, PA 19564, USA \\ ${ }^{\mathfrak{c}}$ Environmental Health / Safety Corporate Ergonomics, Raytheon, 6620 Chase Oaks Blvd, Bldg 4 MS 8524, \\ Plano, TX 75266, USA
}

\begin{abstract}
Process improvement initiatives are receiving renewed attention by large corporations as they attempt to reduce manufacturing costs and stay competitive in the global marketplace. These initiatives include 5S, Six Sigma, and Lean. These programs often take up a large amount of available time and budget resources. More often than not, existing ergonomics processes are considered separate initiatives by upper management and struggle to gain a seat at the table. To effectively maintain their programs, ergonomics program managers need to overcome those obstacles and demonstrate how ergonomics initiatives are a natural fit with continuous improvement philosophies.
\end{abstract}

Keywords: lean, continuous improvement, ergonomics, integration, process

\section{Introduction}

This technical presentation will review the challenges faced by three experienced ergonomics professionals as they work with large U.S. based corporations to integrate ergonomics into continuous improvement initiatives. The benefits of process improvement are well understood and widely recognized by managers and engineers within manufacturing. More often than not, those same manufacturing organizations often have long term ergonomics processes that have made progress in areas of worker safety, injury reduction, and design for manufacturability. However, the ergonomics process is most often housed within the safety and health department and not considered by engineering and management as a necessary participant as they move forward with continuous improvement initiatives.

Continuous improvement and ergonomics philosophies share many common goals including worker safety, waste reduction, and process improvement. In order to efficiently and effectively implement successful programs, it is vital that corporations eliminate housing the initiatives in separate silos each with its own performance measures, injury reduction goals, anticipated pay-backs, and return-on-investment. By combining efforts, job analysis, and data collection strategies, the time to document issues can be reduced enabling limited resources to be focused on solution identification, implementation, and validation. This article provides case study examples of strategies that have been used to integrate ergonomics and continuous improvement within several large US corporations. Methods for overcoming obstacles, selling to management, and issues related to standardization and sustainability are discussed.

\section{Effective ergonomics processes}

Six core elements have been documented as critical for the success of an ergonomics program (GAO, NIOSH 1997). Each of these core elements is briefly described below along with how that element ties in with continuous improvement initiatives (Monroe, 2006). ${ }^{*}$

\subsection{Management commitment}

Management provides staffing and funding resources for ergonomic initiatives. Management ensures that ergonomic efforts align with both site and

\footnotetext{
* Corresponding author. kmergonomics@aol.com
} 
corporate goals and strategies including process improvement. Their involvement has the psychosocial benefit of demonstrating to employees the importance of safety and health within the organization.

Management is able to require accountability for ergonomics at all levels of the organization. This ensures that ergonomics abatement is a priority as engineers move forward with continuous improvement efforts.

\subsection{Employee involvement}

Employees know their jobs better than anyone else. Trained employees can assist in analyzing jobs and identifying waste in the process. Employee involvement in solution development and implementation increases the likelihood that new tools and redesign modifications will be well received and utilized as designed. Employees can also assist with training other employees on best practices and techniques that reduce physical stress when performing job tasks.

\subsection{Identification of problem jobs}

Ergonomics programs traditionally have looked to lagging indicators to first identify problem jobs. This includes workers' compensation records, incident investigations, and employee complaints. Other historical data related to process issues such as downtime and quality can be used to identify trends and identify current issues that require immediate attention.

\subsection{Analyzing and developing controls for problem jobs}

Validated ergonomic job analysis tools can provide benefits beyond the initial application to identify and quantify ergonomic risk factors. They can also assist in evaluating the impact proposed solutions will have on the process. This includes accounting for the characteristics of the worker population as well as evaluating the effectiveness of workstation redesign options.

Ultimately, ergonomics can be incorporated as an integral part of the engineering design process allowing for a proactive approach to ergonomics design and eliminating the waste associated with a traditional reactive approach to ergonomic issues. Engineers can then conduct a cost saving analysis as applicable to demonstrate the impact that the ergonomic elements of their project have had on the bottom line.
Specifically, increased productivity and quality can be tracked and quantified as a tracking metric.

\subsection{Training and education}

Training should be conducted across all levels of the organization. The content and length of training will vary for different groups as appropriate for their role in the process. The training should be customized to the specific site work environment, continuous improvement methodology, and site specific examples and workshops. This will increase participant retention of knowledge and the effectiveness of the training program.

\subsection{Medical management}

Ergonomic related injuries develop over time and are cumulative in nature. The number and severity of injuries can be minimized by encouraging early reporting and intervention. This is a natural fit with process improvement initiatives.

\section{Carpenter}

Carpenter Technology Corporation is a global manufacturer of specialty steel products. At its Reading, PA, USA location there are over 1,000 hourly employees.

\subsection{Background}

The current formal ergonomics initiative has been in place at the Reading plant since 2008 when the number of injuries associated with musculoskeletal risk factors was deemed unacceptable by management, including executive leadership. At that time, the decision was made to dedicate resources for the development of a detailed ergonomics process under the direction of the Health and Safety Department. Simultaneously, efforts were underway by the Quality staff to initiate a formal $5 \mathrm{~S}$ program. Over the next three years the Ergonomics and $5 \mathrm{~S}$ initiatives worked separately from one another, both with varying degrees of success. It was not until the full weight of numerous organizational initiatives, including Ergonomics and 5S, was felt by Production that dialog began about the need to streamline programs. 


\subsection{Ergo lean}

In the interest of "leaning" the various implemented processes, opportunities to integrate Ergonomics and $5 \mathrm{~S}$ were identified and pilot cases were initiated. In addition to efficiencies realized on the production floor (i.e. one work analysis, one interview with operators, one interruption to operations), the breaking down of organizational silos also was observed. Borrowing from the medical rehabilitation model in which an interdisciplinary approach to patient care is the standard, the benefits of an integrated approach to this manufacturing organization are just beginning to be recognized. Of greatest interest, is the methodology for performing an integrated risk assessment which concurrently evaluates ergonomics (safety), productivity, and quality, and begins working on integrated solutions that consider the effects on both up and down stream internal customers.

\section{Raytheon}

\subsection{Ergonomics and design-in strategies}

Raytheon has had a strong ergonomics program since the 1980's. The transition to a more proactive, engineering design based process has been more difficult to sustain. In 2005 Raytheon implemented a proactive risk assessment process for both manufacturing personnel and computer users. This definitely helped Raytheon turn a corner on efforts to eliminate ergonomics risk from the workplace using a prioritization tools. To increase the emphasis on design-in ergonomics, the Health/Safety Ergonomics team teamed up with the Human Factors / Engineering group to establish integrated design process tools such as a workspace planning tool, workstation design checklist, and manual transport analysis protocol. A human performance modeling and simulation plan is still in development.

\subsection{Ergonomics and lean}

When asked to participate in the Raytheon Lean Scorecard Initiative, we took full advantage. Safe- ty/ergonomics is just one of eight scorecard designed to assess practices and behaviors that lead to improved quality, safety, productivity, and competitive advantage. This annual assessment helps manufacturing areas establish a continuous improvement roadmap that is sustainable and a more seamless ergonomically designed workplace.

\section{Summary}

Tying ergonomics in with site continuous improvement processes is the best way to ensure a long term sustainable ergonomics process. It leverages limited resources and makes the best use of employee and management time. Ergonomics program managers may need to develop a "sales pitch" to initially get management support for their active involvement in continuous improvement initiatives. Once there, they can build awareness by demonstrating the success of ergonomics in a few key projects. Over time, ergonomics will become an integral part of both the site culture and the continuous improvement process.

\section{References}

[1] The US General Accounting Office (GAO). 1997 Report Worker Protection: Private Sector Ergonomics Yield Positive Results. CDC Report. 1997.

[2] National Institute of Occupational Safety and Health (NIOSH) Elements of Ergonomics Programs: A Primer Based on Workplace Evaluations of Musculoskeletal Disorders. NIOSH Publication No. 97-117. March, 1997.

[3] Monroe, K.A. Ergonomics: Revisiting the Basics. Industrial Engineer, Volume 38, No 3. 2006.

[4] Walder, J, J. Karlin, and C. Kerk. Integrated Lean Thinking \& Ergonomics: Utilizing Material Handling Assist Device Solutions for a Productive Workplace. A Material Handling Industry of America (MHIA) White Paper. November 1, 2007.

[5] Liker JK. The Toyota Way. New York: McGraw-Hill, 2003.

[6] Department of Health and Human Services. Ergonomic Guidelines for Manual Material Handling. NIOSH Publication No. 2007-131.

[7] Fabrizio TA and Tapping D. 5S for the Office: Organizing the Workplace to Eliminate Waste, Productivity Press, New York City, 2006. 